European Journal of Environment and Public Health

2019, 3(2), em0028

ISSN: 2468-1997

\title{
Cultural Competency-Related Content among Florida Colleges of Pharmacy Curricula
}

\author{
Jason T. Schales ${ }^{1}$, Gebre-Egziabhe Kiros ${ }^{1}$, Fran T. Close ${ }^{1}$, Rima Tawk ${ }^{1 *}$ \\ ${ }^{1}$ Institute of Public Health College of Pharmacy and Pharmaceutical Sciences Florida A\&M University Tallahassee, FL, \\ USA
}

\section{*Corresponding Author: rima.tawk@famu.edu}

Citation: Schales, J. T., Kiros, G.-E., Close, F. T. and Tawk, R. (2019). Cultural Competency-Related Content among Florida Colleges of Pharmacy Curricula. European Journal of Environment and Public Health, 3(2), em0028. https://doi.org/10.29333/ejeph/5867

Published: July 31, 2019

\section{ABSTRACT}

Objective. Assess cultural competency-related content in Pharmacy curricula among Colleges/Schools of Pharmacy in the state of FL.

Methods. Content analysis was used to examine PharmD course descriptions among 5 FL accredited Colleges of Pharmacy. All course descriptions were obtained through the institutes' websites and the Florida Department of Education's Statewide Course Numbering System. The text query analysis gathered and compared key terms among all universities using QSR NVivo11 software. In addition, the association between the number of courses with cultural competency-related content for each PharmD program and each class year were examined.

Results. Cultural competency related content differed by PharmD programs and class years. Only first-year (P1) pharmacy students showed significant differences in exposure to courses with cultural competencyrelated content.

Conclusion. This study implies PharmD education curricula needs to incorporate more exposure to cultural competency-related content within Pharmacy programs. Implementing cultural competence training would equip pharmacists in delivering competent care to vulnerable patient populations.

Keywords: cultural competency, health disparities, vulnerable populations, PharmD curricula

\section{INTRODUCTION}

Cultural competence is a set of congruent behaviors, attitudes, and policies that come together in a system, agency, or among professionals and enable that system, agency, or those professionals to work effectively in crosscultural situations (Cross, Benjamin and Isaacs, 1989). The Accreditation Council for Pharmacy Education (ACPE) mandated cultural competency training in the Doctor of Pharmacy (PharmD) curricula to prepare candidates to practice in culturally diverse environments (2015). This mandate indicates a major shift in Pharmacy core curriculum, both presently and increasingly in the future (Zweber, 2002). Pharmacists are essential members of health care teams, as they are not only involved in delivering high-quality patient care but also in decreasing disparities in pharmaceutical therapy (e.g., disparities in prescriptions, treatments, access to medications, adherence, and response to pharmaceuticals) (Echeverri, Brookover and Kennedy, 2010, 2011). Improving cultural competencies within Pharmacy curricula can reduce such disparities. 
Beginning in the 1950s, nursing professionals have been the pioneers in cultural competency education (Allen, 2010). As early as 1983, the National League of Nursing considered race, ethnic, culture, and diversity criteria for nursing curricula (DeSantis and Lipson, 2007). In 1986, the first cultural diversity guideline for nursing education was proposed by the American Nurses Association (Hunter and Krantz, 2010). It was not until the early 1990's the profession of pharmacy began recognizing the need for culturally sensitive health care practitioners (O'Connell et al., 2013). Ten years later, cultural sensitivity gained momentum and finally was incorporated into Pharmacy curriculum (O'Connell et al., 2013).

Although greater emphasis on cultural competency has been incorporated into pharmacy curricula in recent years, the range of content remains difficult to measure and is inconsistent (O'Connell et al., 2013). Information on content depth, as well as impact on learning and practice of such programs is just beginning to be distributed. Nearly 20 years ago, 50\% of pharmacy schools did not include minority health issues in their curriculum (De Bittner and Monsanto, 1992). In addition, the first pharmacy textbook on culturally competency was not published until 2008 (Halbur and Halbur, 2008).

Florida (FL) currently recognizes six Pharmacy colleges/schools accredited by the ACPE. These colleges/schools include University of Florida (UF), Florida Agricultural \& Mechanical University (FAMU), University of South Florida (USF), Nova Southeastern University (NSU), Palm Beach Atlantic University (PBAU), and Larkin Health Sciences Institute College of Pharmacy. University of Florida (UF) is FL's first public school of Pharmacy, established in 1925 (University of Florida, 2018). Florida Agricultural \& Mechanical University (FAMU), a "Historically Black College", is FL's second public school of Pharmacy established in 1951 (Florida Agricultural and Mechanical University, 2016). Nova South Eastern University (NSU) was FL's first private school of Pharmacy founded in 1987 and the state's first school of Pharmacy in the Southern region of FL (Nova Southeastern University, 2018).

With nearly $50 \%$ of FL's population identifying as minorities due to a shift in its demographics (US Census Bureau, 2017), it is essential our future pharmacists are culturally knowledgeable. According to the literature, there are limited published studies assessing the content of cultural competencies within FL colleges of Pharmacy (Assemi, Cullander and Hudmon, 2004; Evans, 2006; Westberg, Bumgardner and Lind, 2005). If certain topics are not being stressed, such as health disparities, vulnerable populations, and cultural competencies within FL's Pharmacy curricula, future pharmacists may be at risk of lacking the knowledge, attitude, and skills in promoting better health for all. Therefore, the purpose of this study is to assess cultural competency-related content in Pharmacy curricula among Colleges/Schools of Pharmacy in the state of FL.

\section{METHODS}

Current colleges/schools of Pharmacy curricula for the 2017-2018 school year were reviewed for the state of FL. A list of 6 accredited schools of Pharmacy was generated from the ACPE website (ACPE, 2017). However, Larkin Health Sciences Institute College of Pharmacy could not be included due to lack of access to course descriptions and curricula. Schools were identified as either public or private universities. University of Florida (UF), Florida Agricultural \& Mechanical University (FAMU), and University of South Florida (USF) were represented as state public colleges of Pharmacy. Whereas Nova Southeastern University (NSU) and Palm Beach Atlantic University (PBAU), were represented as state private colleges of Pharmacy.

For each of the 5 colleges/schools identified, current program curricula data were obtained through the schools' website. The Florida Board of Education's Course Numbering System was utilized to obtain course descriptions for all public colleges. For FL's private universities, their course descriptions were made available on their schools' website. Undergraduate programs and standalone Bachelor of Science in Pharmaceutical Sciences programs were excluded from the analyses. Further, all course electives, and outside requirements (such as Introductory Pharmacy Practice Experience (IPPE), Advanced Pharmacy Practice Experience (APPE), Inter-professional Education (IPE), Continuing Professional Development (CPD), volunteering, case studies, etc.) were excluded as well.

For the content analysis, an exploratory text query analysis was conducted (Creswell, 2013). The course title and course descriptions were mapped using QSR NVivo11 software to graphically display the curricula to indicate which competencies and associated domains were relative to each other. Any word of phrase that dealt with cultural competencies was included. Following identifications of entries in the descriptions, cultural competency-related content was also influenced by Public Health Core Values (Commissioned Corps of the U.S. Public Health Service, n.d.), the Online Journal of Cultural Competence in Nursing and Healthcare (Online Journal of Cultural Competence in Nursing and Healthcare, 2011), as well as the Robert Wood Johnson Foundation Study (RWJF) developed by Robert C. Like (Like, 2001).

Key terms were identified, but not limited to, skills, attitude, critical thinking, quality of life, minority health, health disparities, perspectives, community health, patient centered care, vulnerable populations, socio-economic status, social determinants, sexual/gender orientation, and prevention. Once terms were identified, they were coded for each university. The terms 


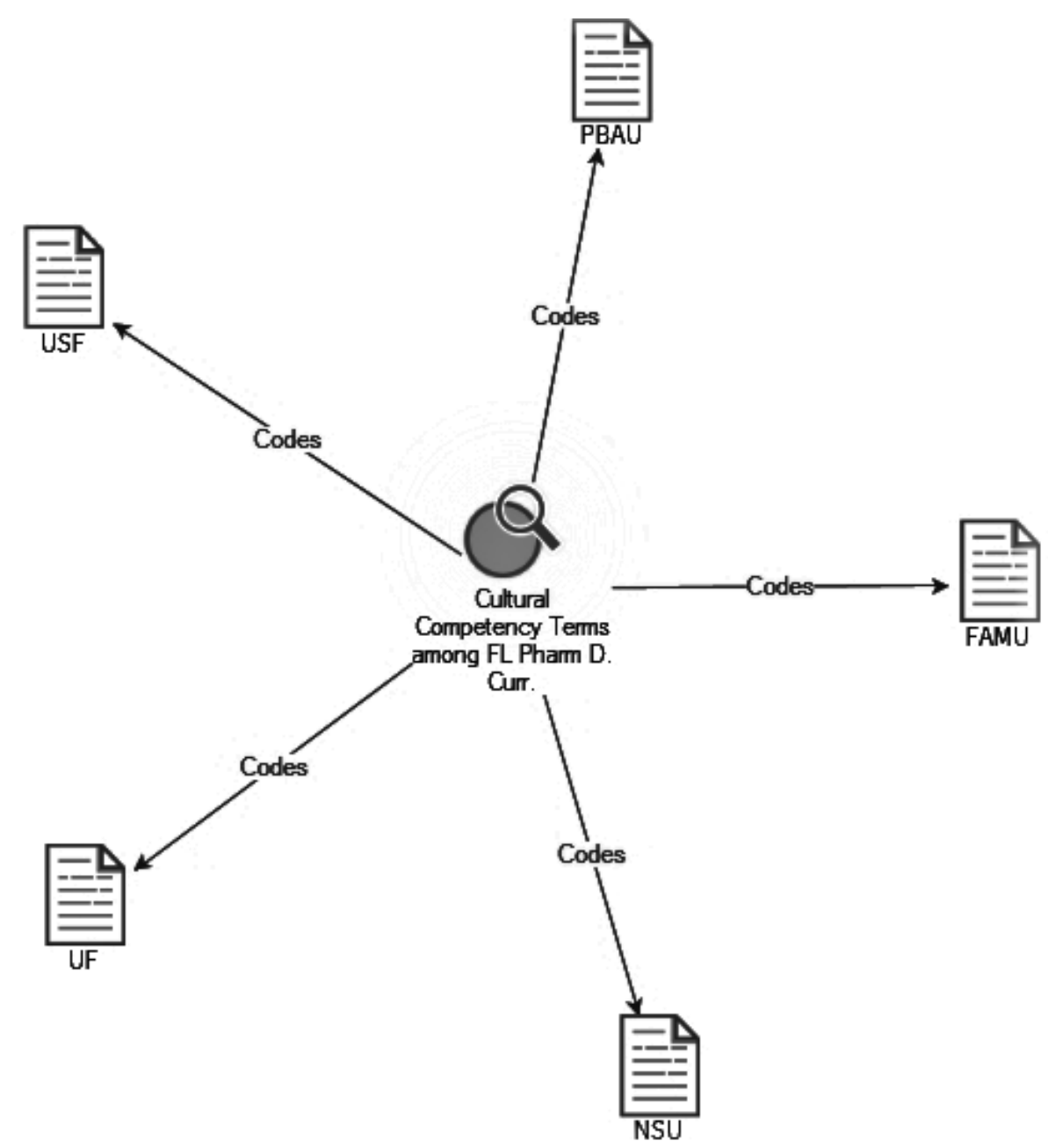

Figure 1. Explore Diagram with Themes Associated with PharmD Programs

identified were arranged to measure the following outcomes: comparison, word frequency and word count. Data collected were cross-checked for completeness. Qualitative findings distinguished courses most geared towards cultural competencies.

The association between the number of cultural competency courses of each PharmD program and the different stages in a pharmacy curriculum (class year) were examined using SAS Version 9.4 (SAS Institute Inc., Cary, NC). Contingency tables were generated per class year (P1 through P4). The statistical test used was Chisquare. Courses were listed in the order in which they occurred in the curriculum to allow for a statistical depiction of the progression of each program. All $\mathrm{p}$ values were interpreted as significant based on an alpha level of 0.05 .

\section{RESULTS}

The content analysis consisted of generating the Explore Diagram. The Diagram presented themes such as cultural competencies, health disparities, vulnerable populations linked to FL PharmD programs (Figure 1). For cultural competencies, content included Affiliation, Attitudes, Beliefs, Comfort in clinical interaction, Diversity, Experiences, Exposure, Interests, Knowledge, Language, Multi-culture, Oppression, Perception, Race/Ethnicity, Religion, Self-awareness, Skills, Training, Tolerance, and V alues. As for health disparities, content grouping consisted of Access to Care, Age, Awareness, Class, Community, Community Affiliation, Disability, Discrimination, Education, Environment, Gender, Harassment, Health Outcomes, Health Risk, Health Status, High Risk, Homosexuality, Hostile Climate, Immigrant health, Inequalities, Inequities, Institutional Racism, Language Barrier, Location, Nutrition/Diet, Preparedness, Prevention, Quality of care, Race, Refugee Status, Religion, Self- Destructive Behavior, Social Determinants, Social Justice, Sexual Orientation, Social Support, Societal Racism, Socio Economic Status, Stigma, Transportation, and Violence. Finally, for vulnerable populations, content classification involved Children, Drug \& Alcohol Addiction, Geriatrics, People living with HIV/AIDS, Homelessness, Incarcerated Populations, Intellectual Disabilities, Lesbian, Gay, Bisexual, Transgender, Queer (LGBTQ), Men who Have Sex with Men (MSM), Mental Health, Military Veterans, Minorities, Persons of Color, Refugees, Religious Affiliations, Sex Workers, Victims of Violence, and Women. 


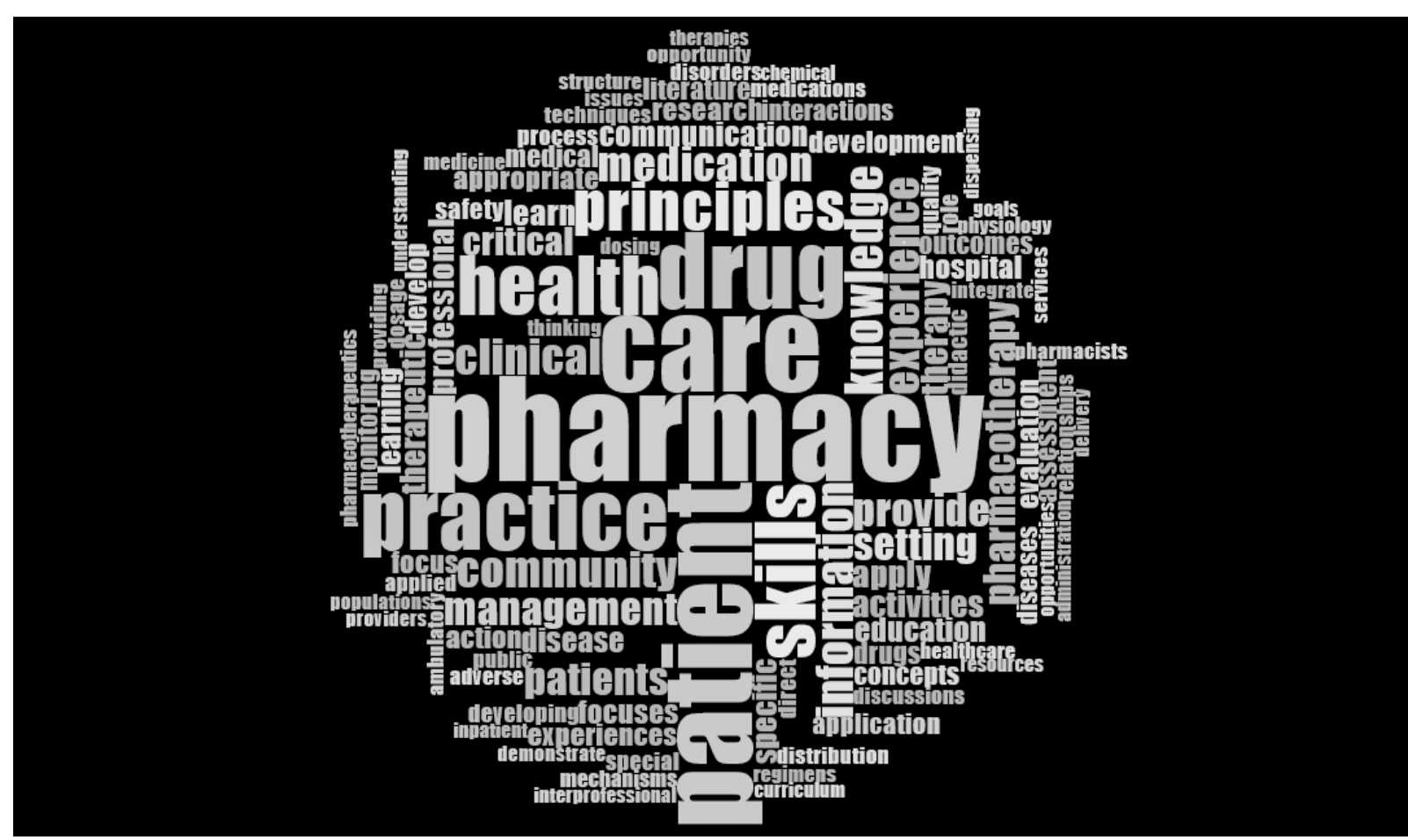

Figure 2. Cultural Competency Language of PhamD Curricula via Word Cloud

Table 1. Distribution of Cultural Competency Courses by Colleges of Pharmacy in Florida

\begin{tabular}{cccc}
\hline & Total Courses (N) & Total CC Courses (n) & Percent \\
\hline FAMU & 47 & 12 & $26 \%$ \\
\hline NSU & 53 & 22 & $42 \%$ \\
\hline PBAU & 45 & 23 & $51 \%$ \\
\hline UF & 40 & 22 & $55 \%$ \\
\hline USF & 42 & 21 & $50 \%$ \\
\hline
\end{tabular}

Table 2. Percentage Distribution of Cultural Competency Courses by Pharmacy Class Year among Colleges of Pharmacy in Florida

\begin{tabular}{ccccccc}
\hline Class Year & FAMU & NSU & PBAU & UF & USF & P-value \\
\hline P1 CC & 7 & 20 & 25 & 56 & 31 & $<\mathbf{0 . 0 5}$ \\
\hline P2 CC & 15 & 40 & 53 & 60 & 54 & .1647 \\
\hline P3 CC & 46 & 36 & 78 & 57 & 50 & .4421 \\
\hline P4 CC & 50 & 75 & 80 & 75 & 83 & 0.7214 \\
\hline
\end{tabular}

Of the 5 Colleges/Schools of Pharmacy, UF reported the most used cultural competency language at approximately $83.9 \%$. At the same time, USF, NSU, and PBAU reported $78.0 \%, 73.5 \%, 71.3 \%$, respectively, while FAMU had the lowest frequency at $60.6 \%$. Among all 5 colleges/schools of Pharmacy; "patient", "practice", "pharmacy", "drug", "health" and "care" were the most frequently used words (Figure 2). Whereas "interprofessional", "structure" and "delivery" were some of the least used words (Figure 2).

With respect to coursework, there was a combined total of 227 courses offered among all FL colleges of Pharmacy (excluding electives). Out of the 227 courses, 100 courses were geared towards cultural competencies. UF reported the highest percentage of courses at 55\%. PBAU included the second highest percentage of courses at $51 \%$, while FAMU had the lowest percentage of courses at only $26 \%$ (Table 1). Furthermore, only year 1 (P1) showed differences in experiences among all universities $(\mathrm{p}<.05)$. There was no statistical significance between year 2 (P2) through year 4 (P4) in exposure with cultural competency courses (Table 2).

\section{DISCUSSION}

The qualitative and the quantitative findings complemented each other and gave a comprehensive picture of culturally competent-related content in Pharmacy curricula. In the qualitative analysis, UF had the highest word frequency percentage, while FAMU reported the lowest word frequency. One interpretation for this finding may be due to other universities providing a more elaborate course description, as compared to FAMU, resulting in a 
higher word frequency percentage. The content of the course description may not accurately reflect the cultural competency related content which may be influenced by teaching approaches, methods and strategies (eg; activities, assignments, etc.). With changes in the demographics of the US population, it is important to prepare Pharmacy graduates to be capable of practicing in a multicultural society (Onyoni and Ives, 2007). With FAMU being the leader in minority education, it is important their program optimizes culturally competent-related content in their course descriptions.

As it pertains to the quantitative analysis, our findings identified differences in cultural competency related content for the P1 class year. However, exposure to cultural competency- related content was not statistically significant for P2, P3, or P4 years. Our findings indicated as PharmD students progressed throughout their program, no differences were captured in the exposure of cultural competency related content. exposures. To our knowledge, such an assessment in exploring cultural competency-related content within Pharmacy curricula using both qualitative and quantitative approaches has not been done before.

Our study has several limitations. First, some PharmD programs did not include their course electives on their websites leading the authors to exclude all electives from the analysis. We believe this exclusion could have altered our results, mainly those electives geared towards cultural competency-related content. Due to the lack in the availability of course objectives, the authors believe those schools' offering fewer courses, relating to culturally competency-related content, are well positioned through the curricula and can be effective in teaching relevant knowledge and skills. Another limitation of the study is not having publicly available syllabi (including courses learning objectives/outcomes) potentially affecting our findings. Hence, only five out of the six accredited colleges of Pharmacy were used. This study does not include all universities. As a result, it is not a true representation of the state. Given the small sample size, implications from the quantitative data are speculative. However, this study does provide us with a general sense on cultural competencies and its involvement with FL PharmD curricula.

Particularly, content analysis built for themes such as "cultural competencies", "health disparities", "vulnerable populations" had many synonyms. We selected only one variation mostly. However, for some terms (e.g., culture and cultural competence), more than one alternative was included. Some terms are progressing or can be undecided such as "sexual minority", "gender minority", or "transgender". As a result, we believe restricting our focus list may have biased our results and prevented us from more evident findings. Further, because such a study only reflects PharmD curricula in the state of FL, this does not serve as a true representation of the entire nation. Some universities may be in the process of revamping their curricula to ensure more cultural diversity and competencies. Such alterations to programs were not considered. A final limitation to the study pertains to the access of the resources based solely on publicly-available web-based data. This restriction may have witheld the presence of courses or course descriptions in sections of schools' websites only available to those with institutional access, or other offerings not advertised online. It is uncertain how website information was frequently updated at these institutions; thus, the dataset may have unintentionally left out existing resources, or included resources that are no longer available.

Dolhun and colleagues (2003) attempted to create a standardized tool for assessing cultural competencies in medical schools. Further, the Association of American Medical Colleges (AAMC) has developed a Tool for Assessing Cultural Competence Training (TACCT) to assist colleges of Medicine in integrating cultural competence content into their curricula (Association of American Medical Colleges, 2005). Used in conjunction with materials that identify optimal educational methods and evaluation strategies, TACCT reflects the input of experts in cultural competence and medical education to provide validated recommendations on curriculum content. Currently, a standardized evaluation tool is not available for use in colleges of Pharmacy.

Considering the lack of instrumentation in assessing cultural competence in pharmacy curricula, Echeverri and colleagues (2010) adapted two instruments to measure the cultural competence of pharmacy students: The Clinical Cultural Competency Questionnaire (CCCQ) (Like, 2001), and the California Brief Multicultural Competency Scale (CBMCS) (Gamst, Dana, Der-Karabetian, et al., 2004). In the authentication process, the CCCQ allowed the identification of nine constructs for curriculum development and the CBCMS confirmed the actuality of four different constructs needed to measure multicultural competence (Echeverri, Brookover, Kennedy, 2010). Although both measures were found to be reliable instruments for pharmacy students, the CCCQ did not include specific domains considered critical when teaching cultural competence, such as awareness of racial dynamics and self-reflection included in the CBMCS (Boutin-Foster, Foster and Konopasek, 2018; Watt, Abbot and Reath, 2016). In contrast, it was decided the CBMCS was too brief to comprehensively evaluate training needs (Echeverri et al., 2018).

An additional study explained how working with various cultural communities within a college's geographical area or state can further develop a health care curriculum geared towards culturally competent patient-centered care (Kamaka, 2010). O'Connell and colleagues (2013) described the tedious process of generating a model curriculum. This paradigm implies defining competencies students should demonstrate at graduation, developing learning objectives, instructional strategies, and evaluation techniques for each learning objective. A recent study 
demonstrated students' self-perception towards cultural competency increasing in terms of knowledge and skills, but not in attitude throughout their program years (Crawford et al., 2016).

O'Connell and colleagues (2013) stress assessments of what incoming Pharmacy students know and perceive about diversity, as well as their experiences, beliefs and expectations, will need to be considered when becoming accustomed to patient-centered care. Assessment on the delivery of cultural competence regarding patient satisfaction, health literacy, medications adherence, continuity of care, health outcomes, health disparities, and health care expenses will be essential. Cultural competence should be included within inter-professional education and training, as it is an expanding model for patient care.

Going forward, including more variables such as course electives, student learning outcomes, and extracurricular activities (IPPE, APPE, IPE, CPD experiences, volunteering/ community outreach, service learnings) would help reduce any bias in outcomes among PharmD programs. In addition, comparing state to national Pharmacy curricula with respect to cultural competencies could help produce more detailed findings by increasing the sample size. Also, this could assist stakeholders in addressing programs lacking cultural competency components. Curricular outcomes included by both ACPE and AACP's Center for the Advancement of Pharmacy Education (CAPE) suggest cultural competency courses need to be merged into all four years of their pharmacy curricula (Accreditation Council for Pharmacy Education, 2015; Echeverri, 2010).

To establish basic standards in cultural competence for Pharmacy graduates, an initial cultural competency certificate needs to be established. This will require pharmacists to attend education or training activities related to current topics on patient-centered care and health disparities annually or bi-annually to maintain their active certificate. This would encourage professionals to stay up to date with current societal and/or cultural trends. In such a "cyber era", we have a platform to reach various populations. With this, it is necessary to utilize mobile devices, social media, E-learning, online communities, blogs, etc. to create a virtual program that encourages exchange of ideas. With regards to Pharmacy, a national curriculum that utilizes new educational technologies to advance discussion, group work, critical thinking, communication skills, and behavioral change is needed to better understand culturally competent patient-centered care.

More research focusing on educational, patient, and health systems outcomes related to cultural competency is vital to justify time invested and associated costs with this type of teaching and learning, especially the development of training resources, and recruiting and maintaining seasoned faculty. Exploring health care cost savings in relation to patient centered care may further elevate the urgency and priority of incorporating cultural competency content into Pharmacy curricula, as well as highlight the need for adequately trained faculty and staff. Additionally, demonstrating cost savings could result in more possibilities for grant funding by larger organizations and institutions (O'Connell et al., 2013). More effort is needed to focus on cultural competence and its evaluating effects on patient-centered care, as well as patient health outcomes.

\section{RECOMMENDATIONS}

It is recommended clinical cultural competency and health-disparity content be incorporated into Pharmacy curricula to ensure Pharmacists are adequately equipped to deliver competent care to the many diverse patient populations they will be serving. Results indicate the importance of considering the students racial composition when developing curriculum in cultural competence. In addition, developing targeted educational interventions for specific groups may fully engage students in addressing health care needs of diverse populations (Smedley, Butler and Bristow, 2004). Cultural competency related content should be taught by public health professionals. This will ensure students are learning from first hand authorities. Although Pharmacists are exposed to various clinical aspects, it is essential we expose them to non-clinical elements of practice as well. It is further implied colleges of Pharmacy should consider the development, maintenance and support of post-graduate residency/fellowship training. By optimizing care and research for underserved populations, we can ensure such professionals are exposed to vulnerable populations on a continuous basis.

\section{CONCLUSION}

Our findings conclude cultural competency and health-disparity-related content are incorporated into the 5 assessed Florida Schools of Pharmacy curricula. There is a growing body of evidence that suggest the use of cultural competency techniques as one of the effective strategies in reducing health disparities (Onyoni and Ives, 2007). Stressing such language and enforcing cultural competency-related content will give our future professionals an advantage. Pharmacy students may not have the opportunity to acquire such skills outside their formal school curriculum. This study implies PharmD education curricula needs to incorporate more exposure to cultural competency-related content within Pharmacy programs. 
Implementing cultural competence training in health care academic programs is still a challenge, especially when students lack motivation to become culturally competent. Students may not value culturally competent services and its impact on patient outcomes and may be unwilling to deal with personal beliefs and stereotypes (MurrayGarcía et al., 2005; Poirier et al., 2009; Snider, 2014). Pharmacy graduates must be cognizant of any barriers that hinder such outcomes and health. The American College of Clinical Pharmacy (ACCP) reviewed pharmacy programs who were incorporating cultural competency curricula and found there is a great need to assess the impact of this training and its educational outcomes, particularly for the provision of patient-centered, culturally sensitive health care (O'Connell et al., 2013).

\section{REFERENCES}

Accreditation Council for Pharmacy Education. (2015). Accreditation standards and guidelines for the professional program in pharmacy leading to the Doctor of Pharmacy degree. Available at: https://www.acpeaccredit.org/pdf/Standards2016FINAL.pdf (Accessed 26 November 2018)

Allen, J. (2010). Improving cross-cultural care and antiracism in nursing education: A literature review. Nurse Education Today, 30(4), 314-320. https://doi.org/10.1016/j.nedt.2009.08.007

Assemi, M., Cullander, C. and Hudmon, K. S. (2004). Implementation and Evaluation of Cultural Competency Training for Pharmacy Students. Annals of Pharmacotherapy, 38(5), 781-786. https://doi.org/10.1345/aph.1d402

Association of American Medical Colleges. (2005). Association of American Medical Colleges (2018). Tool for Assessing Cultural Competence Training (TACCT) - Initiatives - AAMC. Association of American Medical Colleges. Available at: https://www.aamc.org/initiatives/tacct/ (Accessed 26 November 2018)

Boutin-Foster, C., Foster, J. C. and Konopasek, L. (2008). Viewpoint: Physician, Know Thyself: The Professional Culture of Medicine as a Framework for Teaching Cultural Competence. Academic Medicine, 83(1), 106-111. https://doi.org/10.1097/acm.0b013e31815c6753

Commissioned Corps of the U.S. Public Health Service. (n.d.). Available at: https://www.usphs.gov/aboutus/mission.aspx

Crawford, S. Y., Awé, C., Tawk, R. H. and Pickard, A. S. (2016). A Cross Sectional and Longitudinal Study of Pharmacy Student Perceptions of Readiness to Serve Diverse Populations. American Journal of Pharmaceutical Education, 80(4), 62. https://doi.org/10.5688/ajpe80462

Creswell, J. W. (2013). Educational research: Planning, conducting, and evaluating quantitative and qualitative research. Delhi, India: PHI Learning Private Limited.

Cross, T. L., Benjamin, M. P. and Isaacs, M. R. (1989). Towards a culturally competent system of care. Washington, D.C.: CASSP Technical Assistance Center, Georgetown University Child Development Center.

De Bittner, M. and Monsanto, H. (1992). Minority health issues contents in pharmacy curricula. American Pharmaceutical Journal of Education, 56.

Desantis, L. A. and Lipson, J. G. (2007). Brief History of Inclusion of Content on Culture in Nursing Education. Journal of Transcultural Nursing, 18(1_suppl). https://doi.org/10.1177/1043659606295497

Dolhun, E. P., Muñoz, C. and Grumbach, K. (2003). Cross-cultural Education in U.S. Medical Schools. Academic Medicine, 78(6), 615-622. https://doi.org/10.1097/00001888-200306000-00012

Echeverri, M. (2010). Seven Focus Areas of Cultural Competency for Future Health Professionals: Results of a pilot program employing concept mapping and other techniques. In National Conference Series on Quality Health Care for Culturally Diverse Populations.

Echeverri, M., Brookover, C. and Kennedy, K. (2010). Nine Constructs of Cultural Competence for Curriculum Development. American Journal of Pharmaceutical Education, 74(10), 181. https://doi.org/10.5688/aj7410181

Echeverri, M., Brookover, C. and Kennedy, K. (2011). Factor Analysis of a Modified Version of the California Brief Multicultural Competence Scale with Minority Pharmacy Students. Advances in Health Sciences Education, 16(5), 609-626. https://doi.org/10.1007/s10459-011-9280-9

Echeverri, M., Unni, E., Harpe, S. E., Kavookjian, J., Alkhateeb, F., Ekong, G. and Law, A. (2018). A Revised Scale of Cultural Competence Curriculum in Pharmacy Education: A Multi-School Validation Trial. American Journal of Pharmaceutical Education, 80(3), 412-421. https://doi.org/10.5688/ajpe6602

Evans, E. (2006). An Elective Course in Cultural Competence for Healthcare Professionals. American Journal of Pharmaceutical Education, 70(3), 55. https:// doi.org/10.5688/aj700355

Florida Agricultural and Mechanical University. (2016). College of Pharmacy. FAMU College of Pharmacy. Available at: http://pharmacy.famu.edu/ (Accessed 26 November 2018)

Gamst, G., Dana, R., Der-Karabetian, A., et al. (2004). Cultural Competency Revised: The California Brief Multicultural Competence Scale, Measurement and Evaluation in Counseling and Development. Measurement and Evaluation in Counseling and Development, 37, 163-183. https://doi.org/10.1080/07481756.2004.11909758 
Halbur, D. and Halbur, K. V. (2008). Essentials of Cultural Competence in Pharmacy Practice. Washington, D.C.: American Pharmacists Association. https://doi.org/10.21019/9781582121130

Hunter, J. L. and Krantz, S. (2010). Constructivism in Cultural Competence Education. Journal of Nursing Education, 49(4), 207-214. https:/ / doi.org/10.3928/01484834-20100115-06

Kamaka M. L. (2010). Designing a cultural competency curriculum: asking the stakeholders. Hawaï medical journal, 69(6 Suppl 3), 31-34.

Like, R. (2001). Clinical Cultural Competency Questionnaire (pre- training version). UMDNJ-Robert Wood Johnson Medical School. Available at: http://rwjms.umdnj.edu/departments_institutes/family_medicine/ chfcd/grants_projects/documents/Pretraining.pdf (Accessed 26 November 2018)

Murray-Garcia, J. L., Harrell, S., Garcia, J. A., Gizzi, E., \& Simms-Mackey, P. (2005). Self-Reflection in Multicultural Training: Be Careful What You Ask for. Academic Medicine, 80(7), $694-701$. https://doi.org/10.1097/00001888-200507000-00016

Nova Southeastern University. (2018). Pharm.D. Entry Level Program - Curriculum | NSU College of Pharmacy. NSU College of Pharmacy. Available at: https://pharmacy.nova.edu/pharmd/curriculum.html (Accessed 26 November 2018)

O’connell, M. B., Bittner, M. R., Poirier, T., Karaoui, L. R., Echeverri, M., Chen, A. M. et al. (2013). Cultural Competency in Health Care and Its Implications for Pharmacy Part 3A: Emphasis on Pharmacy Education, Curriculums, and Future Directions. Pharmacotherapy: The Journal of Human Pharmacology and Drug Therapy, 33(12). https://doi.org/10.1002/phar.1353

Online Journal of Cultural Competence in Nursing and Healthcare. (2011). About the OJCCNH. Available at: http://www.ojccnh.org/ (Accessed 26 November 2018)

Onyoni, E. M. and Ives, T. J. (2007). Assessing Implementation of Cultural Competency Content in the Curricula of Colleges of Pharmacy in the United States and Canada. American Journal of Pharmaceutical Education, $71(2), 24$. https://doi.org/10.5688/aj710224

Poirier, T. I., Butler, L. M., Devraj, R., Gupchup, G. V., Santanello, C. and Lynch, J. C. (2009). A Cultural Competency Course for Pharmacy Students. American Journal of Pharmaceutical Education, $73(5), 81$. https://doi.org/10.5688/aj730581

Smedley, B. D., Butler, A. S. and Bristow, L. R. (2004). In the Nation's Compelling Interest: Ensuring Diversity in the Health Care Workforce. Washington, DC: The National Academies Press.

Snider, M. (2014). Cultural diversity: international perspectives, impacts on the workplace and educational challenges. New York: Nova.

University of Florida. History. (2018). College of Pharmacy. Available at: https://pharmacy.ufl.edu/thecollege/college-facts/history/ (Accessed 26 November 2018)

US Census Bureau. (2017). Census.gov. Census Bureau QuickFacts. Available at: https://www.census.gov/ (Accessed Retrieved 26 November 2018)

Watt, K., Abbott, P. and Reath, J. (2016). Developing cultural competence in general practitioners: An integrative review of the literature. BMC Family Practice, 17(1). https://doi.org/10.1186/s12875-016-0560-6

Westberg, S. M., Bumgardner, M. A. and Lind, P. R. (2005). Enhancing Cultural Competency in a College of Pharmacy Curriculum. American Journal of Pharmacentical Education, 69(5), 82. https://doi.org/10.5688/aj690582

Zweber, A. (2002). Cultural competence in pharmacy practice. American Pharmaceutical Journal of Education, 66, 172. 